\title{
Características Morfométricas de los Discos Articulares de la Rodilla en Imágenes de Resonancia Magnética de Mujeres Colombianas
}

\author{
Morphometric Features of the Knee Menisci in Magnetic Resonance Images of Colombian Female
}

Lessby Gómez ${ }^{1,2}$; Carlos A. Diaz ${ }^{3}$ \& Gloria Patricia Baena-Caldas ${ }^{4,5}$

GÓMEZ, L.; DIAZ, C. A. \& BAENA-CALDAS, G. P. Características morfométricas de los discos articulares de la rodilla en imágenes de resonancia magnética de mujeres colombianas. Int. J. Morphol., 39(1):32-37, 2021.

RESUMEN: La población femenina de adultos mayores suele presentar daños de los discos o meniscos articulares de la rodilla (DAR) generalmente derivados de procesos degenerativos de cartílago ocurridos en osteoartritis. Conocer patrones morfológicos estándares de los DAR en una población demográfica específica permite tener claridad de las características autóctonas de dicha población. Una forma de caracterizar estos patrones es a través del diseño de Modelos de Elementos Finitos (MEF) que permiten predecir anormalidades clínicas y radiológicas. Hasta el momento es escasa la evidencia sobre las características morfométricas de los DAR en la población colombiana. Por esta razón, el objetivo del presente estudio fue describir la morfometría de DAR en imágenes por resonancia magnética (IRM) de mujeres colombianas saludables. En este estudio se tomaron 25 IRM de la rodilla de mujeres colombianas con normo-peso, sin antecedentes de patología de la rodilla, ni historia de trauma o cirugía de esta, y con edades entre los 18 y 60 años. El análisis del ancho del cuerno posterior del DAR medial $(12,73 \pm 2,42)$ comparado con el lateral $(8,04 \pm 1,52)$ arrojó diferencia estadísticamente significativa $(\mathrm{p}<0,05)$. Por otra parte, el DAR medial presentó un diámetro antero-posterior aumentado $(38,52 \pm 2,71)$ en comparación con el del DAR lateral $(29,18 \pm 2,75)$ cercano a la significancia estadística $(\mathrm{p}=0.051)$. De igual manera, la altura del cuerno anterior se encontró aumentada en el DAR medial $(4,42 \pm 0,79)$ en comparación con el lateral $(3,66 \pm 0,76)$, con tendencia a la significancia estadística ( $\mathrm{p}=0,063)$. Anatómicamente se reconoce que los DAR medial y lateral se adaptan a la forma y tamaño de los cóndilos femorales, lo que concuerda con las medidas resultantes de la población estudiada, donde se evidencia un DAR medial más largo (diámetro antero-posterior) y menos alto que el lateral.

PALABRAS CLAVE: Anatomía; Discos articulares; Meniscos; Rodilla; Imagen por resonancia magnética.

\section{INTRODUCCIÓN}

La región genicular o rodilla es un complejo sistema articular compuesto por estructuras óseas, cartilaginosas, ligamentosas, vasculonerviosas, las cuales en procesos normales de la marcha reciben considerables fuerzas de compresión. Esto puede contribuir a que la región genicular sufra afecciones en sus componentes anatómicos, principalmente en la edad adulta mayor.

Los discos articulares de la rodilla (DAR), también conocidos como meniscos (palabra derivada del griego por su forma de luneta o media luna) (Fox et al., 2012), son estructuras fibrocartilaginosas que contribuyen a aumentar la concavidad de los cóndilos tibiales para recibir adecuadamente a los femorales. El medial es semicircular, mientras que el late- ral es casi circular. Ambos DAR presentan un cuerno anterior y uno posterior ubicados adyacentes a la eminencia intercondílea del hueso tibia, un margen interno agudo o cortante, un margen periférico grueso, una superficie superior cóncava en contacto con los cóndilos femorales y una inferior casi plana en contacto con los cóndilos tibiales (Chatain \& Bustamante, 1986).

El DAR medial cubre aproximadamente el $60 \%$ de la superficie articular del cóndilo medial de la tibia (Smigielski et al., 2015), por su parte, el DAR lateral es más pequeño, uniforme y móvil, per se a su menor tamaño, ocupa del 60 al $80 \%$ de la superficie articular del cóndilo lateral de la tibia (Fox et al., 2015). Ambos DAR están altamente especializa-

\footnotetext{
${ }^{1}$ Programa de Medicina, Facultad de Salud, Universidad Libre, Cali, Colombia. Grupo Esculapio.

${ }^{2}$ Escuela de Rehabilitación Humana, Facultad de Salud, Universidad del Valle, Cali, Colombia.

${ }^{3}$ Facultad de Salud, Universidad Libre, Cali, Grupo Esculapio, Colombia.

${ }^{4}$ Departamento de Morfología, Escuela de Ciencias Básicas, Facultad de Salud, Universidad del Valle, Cali, Colombia.

${ }^{5}$ Candidato a Doctor en Ciencias Biomédicas. Facultad de Salud, Universidad del Valle, Cali, Colombia.

Subvencionado por: Convocatoria Interna de proyectos de investigación con proyección social 2018 de la Universidad Libre. Código Interno: CIFCS2018-JUN-07-016.
} 
dos en la absorción de la energía producida como resultado de recurrentes cargas (tanto compresivas como cortantes) a las que constantemente está sometida la región genicular.

La forma y el tamaño de los DAR, puede variar de una población a otra, por lo que es importante tener parámetros de referencia en cada población que sirvan para establecer criterios de normalidad (Peters et al., 2018). A la fecha no se conocen estudios de este tipo en la población colombiana, mientras que, a nivel latinoamericano solo se ha encontrado el reporte de estudios en la población chilena y brasileña (Torrez et al., 2011; Braz \& Silva, 2017). La ausencia de estudios dirigidos a establecer parámetros de normalidad en la población colombiana conduce a que las mediciones utilizadas en esta población sean extrapoladas de otras poblaciones como las americanas y europeas, las cuales pueden llegar a ser morfológicamente diferentes.

Generalmente las descripciones morfométricas de los DAR han sido reportadas a partir de mediciones en cadáveres y en resonancia magnética (RM). Esta última corresponde al método más referenciado, siendo empleado para hacer mediciones desde sus imágenes o mediante la construcción de estructuras tridimensionales a partir de procesos de segmentación, como sucede en el desarrollo de modelos de elementos finitos (MEF).

El MEF es un método matemático basado en la solución de ecuaciones diferenciales, que permite modelar diversos tipos de materiales y analizar su comportamiento dinámico en distintas áreas de estudio como lo son la ingeniería y las ciencias de la salud (Vélez et al., 2009). En esta última, los materiales que usualmente se modelan son los tejidos conectivos propios fibrosos (ej. los ligamentos articulares) (Orozco et al., 2018) y los tejidos conectivos especializados cartilaginosos (ej. los discos articulares) (Luczkiewicz et al., 2016). Durante el proceso de elaboración del MEF se debe construir la geometría del material a estudiar, utilizando un software que funciona como una herramienta de simulación de casos hipotéticos de carga para la predicción de posibles condiciones clínicas, tales como la osteoartrosis (Klets et al., 2018). El MEF debe ser parametrizable con el fin de reproducir de manera confiable los rasgos intrínsecos de un individuo, para permitir obtener estructuras con características morfo-funcionales cercanas a la realidad.

El objetivo del presente trabajo fue realizar una caracterización morfométrica de los DAR de mujeres colombianas sanas a partir de imágenes de RM. Esta caracterización aportará variables cuantitativas útiles para el diseño de MEF que permitirá predecir en la población colombiana el riesgo individual de desarrollar patologías en los DAR, como sucede en la osteoartrosis que se presenta en mayor prevalencia en mujeres (Jiang et al., 2017; Kontio et al., 2017; Sun et al., 2019).

\section{MATERIAL Y MÉTODO}

En el presente estudio descriptivo, observacional, de corte transversal, se tomaron 25 imágenes de resonancia magnética (IRM) de la rodilla de mujeres colombianas con normopeso y con edades comprendidas entre 18 y 60 años, que aceptaron voluntariamente hacer parte del estudio. Se incluyeron en el estudio participantes sin antecedentes de dolor o patología de rodilla, ni historia previa de trauma o cirugía en la misma, y se aplicaron los criterios de exclusión establecidos por Bloecker et al. (2012) para determinar normalidad de los meniscos en participantes saludables.

Para la adquisición de las IRM cada participante del estudio fue ubicada en posición supino, con las rodillas naturalmente extendidas. Las 25 IRM de rodilla fueron tomadas en los planos sagital y coronal en la secuencia fs T2 iso 320 (ST 0.6, SL -123.29, RT 1200 ET: 28, FS 3) con cortes cada $1 \mathrm{~mm}$, con un resonador Magneton Skyra Maximize 3T (Siemens, Alemania) de un centro radiológico de la ciudad de Cali-Colombia. Las IRM fueron revisadas por la investigadora principal en colaboración con un especialista en radiología (quienes cuentan con más de 10 años de experiencia trabajando con alteraciones de la marcha), para establecer la calidad de la imagen radiológica y la normalidad articular acorde a los criterios de Kellgren Lawrence (Kohn et al., 2016).

Este trabajo hace parte de un macroproyecto titulado "Estimación del riesgo de osteoartrosis de rodilla, mediante el desarrollo de un modelo de elementos finitos parametrizable que considere la individualidad anatómica y biomecánica del miembro inferior", el cual fue avalado por el Comité de Evaluación Ética y Científica para la Investigación en Seres Humanos de la Universidad Libre.

En cuanto a la cuantificación morfométrica de los DAR, se tomaron algunas de las medidas propuestas por Erbagci et al. (2004): 1) Altura de los cuernos anterior y posterior del DAR lateral en el plano sagital, 2) Altura de los cuernos anterior y posterior del DAR medial en el plano sagital, 3) Ancho de los cuernos anteriores en el plano sagital de los DAR medial y lateral, 4) Altura de los DAR medial y lateral en la porción medial del cuerpo en el plano coronal, 5) Ancho de los DAR medial y lateral en la porción medial de cuerpo en el plano coronal. Las medidas fueron tomadas en el punto más alto de cada cuerno y cuerpo de los DAR en los mencionados planos (Fig. 1). 


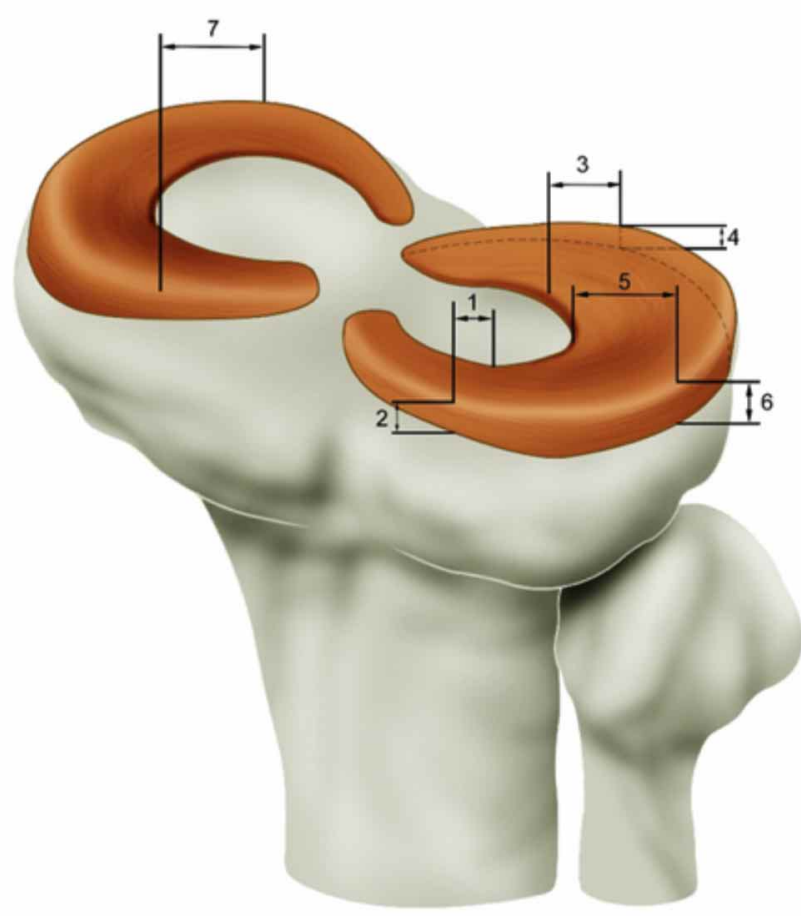

Para el análisis estadístico se determinaron el promedio, la desviación estándar, la mediana de las medidas de los DAR medial y lateral, y se compararon los resultados mediante una prueba de $t$ de student para muestras independientes con un nivel de significancia estadística de $\mathrm{p} \leq 0,05$, empleando el software SSPS versión 24 (IBM Corp., USA).

Fig. 1. Vista posterior de los huesos tibia y fíbula derecha, donde se observan los discos articulares de la rodilla (DAR) medial y lateral ubicados encima de sus correspondientes caras articulares medial y lateral de la tibia. Sobre el DAR lateral fueron representadas con flechas horizontales de dos cabezas las longitudes medidas en ambos DAR, siendo las medidas 1, 3, 5 y 7 correspondientes a las longitudes del ancho del cuerno posterior, del cuerno anterior, del cuerpo y del diámetro antero-posterior de cada DAR, respectivamente. Por otro lado, las longitudes de altura fueron representadas con flechas verticales de dos cabezas (medidas 2, 4 y 6) con las que se midieron las alturas de los cuernos posterior, anterior y del cuerpo de cada DAR, respectivamente.

Tabla I. Valores en milímetros (mm) de las medidas de los discos articulares de la rodilla (DAR), (n=25). CP: Cuerno posterior, CA: Cuerno anterior, C: Cuerpo, A-P: Antero-posterior DS: Desviación estándar, M: Medial, L: Late-

\begin{tabular}{|c|c|c|c|c|c|c|c|c|c|c|c|c|c|c|}
\hline \multirow{2}{*}{ Variables } & \multicolumn{4}{|c|}{ Cuerno posterior } & \multicolumn{4}{|c|}{ Cuerno anterior } & \multicolumn{4}{|c|}{ Cuerpo } & \multirow{2}{*}{\multicolumn{2}{|c|}{$\begin{array}{c}\text { Diámetro A- } \\
\mathrm{P}\end{array}$}} \\
\hline & \multicolumn{2}{|c|}{ Ancho } & \multicolumn{2}{|c|}{ Alto } & \multicolumn{2}{|c|}{ Ancho } & \multicolumn{2}{|c|}{ Alto } & \multicolumn{2}{|c|}{ Ancho } & \multicolumn{2}{|c|}{ Alto } & & \\
\hline DAR & $\mathrm{M}$ & $\mathrm{L}$ & $\mathrm{M}$ & $\mathrm{L}$ & $\mathrm{M}$ & $\mathrm{L}$ & $\mathrm{M}$ & $\mathrm{L}$ & $\mathrm{M}$ & $\mathrm{L}$ & $\mathrm{M}$ & $\mathrm{L}$ & M & $\mathrm{L}$ \\
\hline 1 & 16,4 & 9,70 & 5,54 & 5,91 & 6,39 & 10,0 & 3,19 & 3,79 & 9,15 & 9,29 & 3,27 & 3,98 & 39,3 & 34,4 \\
\hline 2 & 14,0 & 7,46 & 5,59 & 4,98 & 8,91 & 8,43 & 4,72 & 2,92 & 9,17 & 9,13 & 4,22 & 5,18 & 37,0 & 29,1 \\
\hline 3 & 16,2 & 8,56 & 7,37 & 7,37 & 8,56 & 8,09 & 5,24 & 4,29 & 12,3 & 9,29 & 5,20 & 7,52 & 39.7 & 34,0 \\
\hline 4 & 13,1 & 9,07 & 4,42 & 5,31 & 9,74 & 10,8 & 5,09 & 3,10 & 6,86 & 9,74 & 4,20 & 5,53 & 39,3 & 28,9 \\
\hline 5 & 11,9 & 8,27 & 4,62 & 6,33 & 7,79 & 9,25 & 2,69 & 4,62 & 5,95 & 11,7 & 4,91 & 5,07 & 39,4 & 28,7 \\
\hline 6 & 11,5 & 9,50 & 5,06 & 5,31 & 7,73 & 7,43 & 4,50 & 4,09 & 7,39 & 11,5 & 4,25 & 4,14 & 43,0 & 27,6 \\
\hline 7 & 15,0 & 9,50 & 5,05 & 5,66 & 8,49 & 8,89 & 4,44 & 3,64 & 9,02 & 8,41 & 5,31 & 5,97 & 42,2 & 29,3 \\
\hline 8 & 14,0 & 7,55 & 4,90 & 5,77 & 9,11 & 8,83 & 5,30 & 2,89 & 10,7 & 10,9 & 4,53 & 3,67 & 42,6 & 28,2 \\
\hline 9 & 12,0 & 10,7 & 6,02 & 3,79 & 11,1 & 8,88 & 4,71 & 5,99 & 6,51 & 8,38 & 4,67 & 3,73 & 40,0 & 31,0 \\
\hline 10 & 13,1 & 5,40 & 4,14 & 4,68 & 9,18 & 4,86 & 4,50 & 2,88 & 11,1 & 12,4 & 3,43 & 3,25 & 36,9 & 23,0 \\
\hline 11 & 9,04 & 5,88 & 4,52 & 4,67 & 8,59 & 6,33 & 3,92 & 2,86 & 9,51 & 10,8 & 3,10 & 2,88 & 33,3 & 25,8 \\
\hline 12 & 11,0 & 7,00 & 3,77 & 4,84 & 8,61 & 6,44 & 4,84 & 3,74 & 11,6 & 10,5 & 3,38 & 4,88 & 38,3 & 25,5 \\
\hline 13 & 9,82 & 9,18 & 4,51 & 6,92 & 12,1 & 9,34 & 4,99 & 4,03 & 6,72 & 8,76 & 4,49 & 5,10 & 39,3 & 29,7 \\
\hline 14 & 14,4 & 6,90 & 5,32 & 5,32 & 7,49 & 8,29 & 2,76 & 2,77 & 9,42 & 8,14 & 3,28 & 5,27 & 37,5 & 31,5 \\
\hline 15 & 6,78 & 9,34 & 3,74 & 6,31 & 13,3 & 12,8 & 4,90 & 3,98 & 8,19 & 8,85 & 3,55 & 3,77 & 36,2 & 34,0 \\
\hline 16 & 13,8 & 7,14 & 4,91 & 8,03 & 8,03 & 9,37 & 4,24 & 4,46 & 8,20 & 8,88 & 4,56 & 6,38 & 39,5 & 31,2 \\
\hline 17 & 13,1 & 7,75 & 4,88 & 4,87 & 9,30 & 9,97 & 5,09 & 3,32 & 13,6 & 6,05 & 4,34 & 8,80 & 41,3 & 28,9 \\
\hline 18 & 12,7 & 11,1 & 4,64 & 8,49 & 8,07 & 8,41 & 4,47 & 4,29 & 10,7 & 10,3 & 4,00 & 4,28 & 36,6 & 29,4 \\
\hline 19 & 15,4 & 7,90 & 5,01 & 6,17 & 7,13 & 10,0 & 5,01 & 3,85 & 11,5 & 10,4 & 3,55 & 5,31 & 41,0 & 30,5 \\
\hline 20 & 15,6 & 8,35 & 5,57 & 5,36 & 7,49 & 8,99 & 4,29 & 4,07 & 8,81 & 8,13 & 4,97 & 6,34 & 41,1 & 28,7 \\
\hline 21 & 13,4 & 7,06 & 5,16 & 5,25 & 6,32 & 8,77 & 4,00 & 2,84 & 11,1 & 10,1 & 4,17 & 4,46 & 35,5 & 29,9 \\
\hline 22 & 11,1 & 5,40 & 3,66 & 4,13 & 8,42 & 6,67 & 4,29 & 2,86 & 10,4 & 13,5 & 2,18 & 4,43 & 38,0 & 26,7 \\
\hline 23 & 8,45 & 7,67 & 4,44 & 3,19 & 7,61 & 7,67 & 4,65 & 3,48 & 11,3 & 8,41 & 2,65 & 4,20 & 35,1 & 26,7 \\
\hline 24 & 14,1 & 8,62 & 4,66 & 6,21 & 6,55 & 9,83 & 5,69 & 3,80 & 10,5 & 7,90 & 3,05 & 4,55 & 38,2 & 30,8 \\
\hline 25 & 12,4 & 6,09 & 5,58 & 3,90 & 6,40 & 4,84 & 2,97 & 2,97 & 6,54 & 8,96 & 3,19 & 3,87 & 32,6 & 25,9 \\
\hline
\end{tabular}




\section{RESULTADOS}

En la Tabla I se resumen las medidas obtenidas de cada una de las variables estudiadas en los DAR medial y lateral, en cada una de las 25 IRM analizadas, donde se aprecia una importante variabilidad intersujetos.

Los promedios de las medidas obtenidas en los DAR medial y lateral se presentan en la Tabla II. El ancho del cuerno posterior del DAR medial se muestra más ancho que el del lateral, con diferencias estadísticamente significativas $(\mathrm{p} \leq 0,05)$. De igual manera, el DAR medial evidenció un diámetro antero-posterior y una altura del cuerno anterior mayor que la del lateral, sin embargo, ambas mediciones evidenciaron tendencia a la significancia estadística.

Tabla II. Valores en milímetros ( $\mathrm{mm}$ ) del promedio, rango y mediana obtenidos de las diferentes variables medidas en los discos articulares de la rodilla (DAR), $(\mathrm{n}=25)$. CP: Cuerno posterior, CA: Cuerno anterior, C: Cuerpo, DS: Desviación estándar, M: Medial, L: Lateral. $(*)$ corresponde a $\mathrm{p} \leq 0,05$. (\#) valor con tendencia a significancia estadística, sin ser realmente significativo.

\begin{tabular}{|c|c|c|c|c|c|c|c|c|}
\hline & \multirow[b]{2}{*}{ DAR } & \multicolumn{3}{|c|}{ Prome dio $(\mathrm{mm}) \pm \mathrm{DS}$} & \multicolumn{2}{|c|}{ Rango (mm) } & \multicolumn{2}{|c|}{ Mediana (mm) } \\
\hline & & M & $\mathrm{L}$ & $\mathrm{p} \leq 0,05$ & M & $\mathrm{L}$ & M & $\mathrm{L}$ \\
\hline \multirow{7}{*}{ 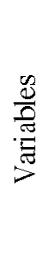 } & CP Ancho & $12,73 \pm 2,42$ & $8,04 \pm 1,52$ & $0,005(*)$ & $6,78-16,40$ & $5,40-11,10$ & 13,10 & 7,90 \\
\hline & CP Alto & $4,92 \pm 0,79$ & $5,55 \pm 1,27$ & 0,773 & $3,66-7,37$ & $3,19-8,49$ & 4,90 & 5,32 \\
\hline & CA Ancho & $8,50 \pm 1,70$ & $8,51 \pm 1,78$ & 0,104 & $6,32-13,30$ & $4,84-12,80$ & 8,42 & 8,77 \\
\hline & CA Alto & $4,42 \pm 0,79$ & $3,66 \pm 0,76$ & $0,063(\#)$ & $2,69-5,69$ & $2,77-5,99$ & 4,50 & 3,74 \\
\hline & C Ancho & $9,45 \pm 2,04$ & $9,62 \pm 1,62$ & 0,717 & $5,95-13,60$ & $6,05-13,50$ & 9,42 & 9,29 \\
\hline & C Alto & $3,94 \pm 0,82$ & $4,61 \pm 1,34$ & 0,550 & $2,18-5,31$ & $2,88-8,80$ & 4,17 & 4,66 \\
\hline & D A-P & $38,52 \pm 2,71$ & $29,18 \pm 2,75$ & $0,051(\#)$ & $32,6-43,0$ & $23,0-34,40$ & 39,30 & 29,10 \\
\hline
\end{tabular}

\section{DISCUSIÓN}

Los DAR son estructuras anatómicas afectadas por patologías como la osteoartrosis y los desgarros meniscales, las cuales en gran medida afectan a mujeres (Jiang et al.; Kontio et al.; Sun et al.). Por tal motivo, el presente estudio establece la magnitud en altura y grosor de los cuernos y cuerpo de los DAR en una muestra de IRM de mujeres colombianas saludables, siendo la única reportada hasta la fecha en dicha población.

Anatómicamente se reconoce que los DAR medial y lateral se adaptan a la forma y tamaño de los cóndilos femorales. En tal sentido, al ser el cóndilo medial más largo y menos convexo que el lateral (Domenech et al., 2003), se esperaría que existieran diferencias significativas entre ambos DAR, tal como se observa en las medidas obtenidas en el presente estudio donde se encontró que el DAR medial evidenció mayor distancia anteroposterior que el lateral y menor altura que este último. Cabe resaltar que estas diferencias solo fueron estadísticamente significativas en el ancho del cuerno posterior, lo cual permitió observar un DAR medial de mayor tamaño.

Las medidas tomadas fueron difíciles de comparar con las reportadas por otros autores, debido a que no existe una estandarización sobre la forma en que debe ser obtenida cada medida. Ello se debe en primer lugar al método empleado para la recolección de la información, que puede dividirse en medidas in vitro, in vivo o in sílico.

Como medidas in vitro se tiene las tomadas en rodillas de cadáveres o en rodillas amputadas (McDermott et al., 2004; Torrez et al.). En estos métodos, aunque la forma de medir puede ser mucho más precisa, presenta como sesgo, las variaciones en medida que pueden derivarse de la deshidratación o la inmersión en sustancias fijadoras.

Las medidas in vivo suelen hacerse de manera indirecta a partir de imágenes de RM o de tomografía axial computarizada (TAC) (Erbagci et al.; Shiang \& Kotecha, 2020). Estos métodos son cada vez más utilizados porque proporcionan información de sujetos vivos y funcionalmente activos. Sin embargo, las medidas pueden tener un error de medición fundamentado en la calidad de la imagen tomada, el tamaño de los cortes de la RM o TAC y la experticia del investigador para el posicionamiento de las marcas de medición, así mismo tienen como limitante que permite tomar medidas en un solo plano (Siorpaes et al., 2012).

Los métodos in sílico se derivan de las imágenes de RM o TAC y permiten la reconstrucción de la estructura en $3 \mathrm{D}$, por lo que permite la toma de diversas medidas (Wenger 
et al., 2013). Sin embargo, el proceso de segmentación es dispendioso y presenta un amplio margen de error, ya que depende de la precisión del investigador en el caso de la segmentación manual o del algoritmo de segmentación en el caso de las automatizadas. Igualmente, este tipo de proceso requiere de un filtro de suavizado que puede modificar la morfometría de la estructura, si no es seleccionado apropiadamente.

Con base en lo anterior, los diferentes artículos encontrados dan cuenta de medidas que son difícilmente comparables como se observa en la Tabla III, hecho que incrementa la complejidad cuando las metodologías de medición son pobremente descritas, por lo que es difícil reproducirlas con exactitud.

Medidas similares a las realizadas en el presente trabajo de investigación, fueron encontradas en diversos estudios que corroboran las diferencias encontradas entre un DAR y otro (Torrez et al.; Erbagci et al.; McDermott et al.; Wenger et al.). En la mayoría de los estudios, independiente del método empleado para tomar la medida y el tipo de población, el cuerpo del DAR lateral resulta ser más ancho y alto que el medial. Cabe destacar que, en el trabajo realizado por Wenger et al., en pacientes con osteoartritis inicial, la altura del cuerpo del DAR lateral fue menor.

Otros estudios que también evaluaron los DAR empleando medidas diferentes, llegaron a conclusiones similares a las halladas en el presente estudio, tal es el caso de Bloecker et al., quienes encontraron que el DAR lateral cubre más área que el medial y que el cuerno posterior presentó mayor grosor que el cuerpo y el cuerno anterior en el DAR medial. Por su parte, Braz \& Silva establecieron que en el DAR medial el cuerno posterior era más ancho que el cuerpo y el cuerno anterior, al tiempo que encontraron diferencias significativas entre el ancho de los DAR lateral y medial, en tres puntos de referencia.

El presente estudio evidenció en la población colombiana un patrón similar en cuanto a la variación de las medidas obtenidas en la población chilena, según lo reportado por Torres et al.; sin embargo, cabe mencionar que los valores reportados en los individuos chilenos fueron relativamente mayores, debido quizás a que la morfometría de ellos se asemeja más a la europea.

Con relación a la aplicación del presente estudio para el desarrollo de MEF parametrizables de los DAR, se logra concluir que de las siete variables cuantificadas solo debería considerarse el promedio de las tres que presentaron variaciones estadísticamente significativas o cercanas a ella, con el fin de ahorrar tiempo y recurso computacional al momento de elaborar el MEF (Guo et al., 2017; Cooper et al., 2019).

\section{AGRADECIMIENTOS}

Al laboratorio de Biomecánica de la Universidad Libre por el uso de sus instalaciones. A Fabián Cabrera Pazmino por su colaboración en el diseño gráfico del presente estudio.

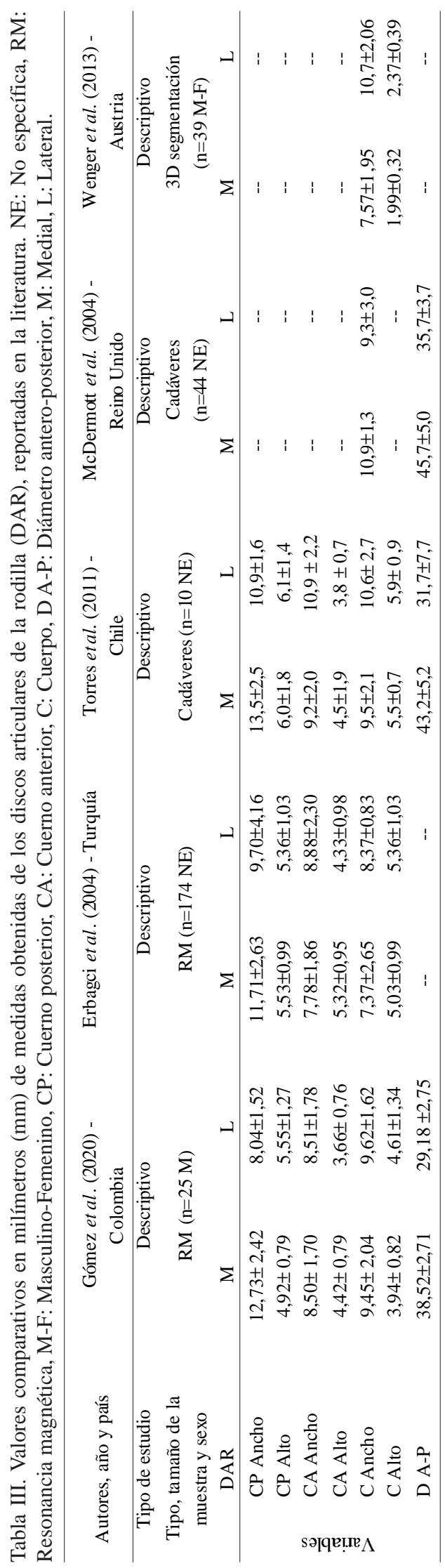


GÓMEZ, L.; DIAZ, C.A. \& BAENA-CALDAS, G. P. Morphometric features of the knee menisci in magnetic resonance images of Colombian female. Int. J. Morphol. 39(1):32-37, 2021.

SUMMARY: The elderly female population usually presents damage to the articular discs or menisci of the knee (ADK), generally derived from degenerative cartilage processes that occur in osteoarthritis. Knowing the standard morphological patterns of ADKs in a specific demographic population allows clarity of the indigenous characteristics of that population. One way to characterize these patterns is through the design of Finite Element Models (FEM) that would enable predicting clinical and radiological abnormalities. So far, there is little evidence on the morphometric characteristics of ADKs in the Colombian population. Thus, the objective of the present study was to describe the morphometry of ADK in magnetic resonance imaging (MRI) of healthy Colombian women. In this study, 25 MRIs of the knee of Colombian women with normal weight were taken, with no history of knee pathology, trauma, or surgery, and with ages between 18 and 60 years. The analysis of the width of the posterior horn of the medial ADK $(12.73 \pm 2.42)$ compared to the lateral $(8.04 \pm 1.52)$ showed a statistically significant difference $(\mathrm{p}<0.05)$. On the other hand, the medial ADK presented an increased anteroposterior diameter (38.52 \pm $2.71)$ compared to that of the lateral $\operatorname{ADK}(29.18 \pm 2.75)$ close to statistical significance $(\mathrm{p}=0.051)$. Similarly, the height of the anterior horn was found to be increased in the medial ADK (4.42 \pm 0.79$)$ compared to the lateral $(3.66 \pm 0.76)$, with a trend towards statistical significance $(\mathrm{p}=0.063)$. Anatomically, it is recognized that the medial and lateral ADK adapt to the shape and size of the femoral condyles, which is in agreement with the results of the studied population where measurements show that medial ADK has a longer (anterior to posterior diameter) and a shorter height than the lateral ADK.

KEY WORDS: Anatomy; Articular discs; Menisci; Knee; Magnetic Resonance imaging.

\section{REFERENCIAS BIBLIOGRAFICAS}

Bloecker, K.; Wirth, W.; Hudelmaier, M.; Burgkart, R.; Frobell, R. \& Eckstein, F. Morphometric differences between the medial and lateral meniscus in healthy men-a three-dimensional analysis using magnetic resonance imaging. Cells Tissues Organs, 195(4):353-64, 2012.

Braz, P. R. P. \& Silva, W. G. Meniscus morphometric study in humans. J. Morphol. Sci., 27(2):62-6, 2010.

Chatain, L. I. \& Bustamante. B. J. Anatomía Macroscópica, Funcional y Clínica. Ciudad de México, SITESA, 1986.

Cooper, R. J.; Wilcox, R. K. \& Jones, A. C. Finite element models of the tibiofemoral joint: A review of validation approaches and modelling challenges. Med. Eng. Phys., 74:1-12, 2019.

Domenech, R. G.; Moreno, M. C. \& Fernandez, V. M. Anatomía y biomecánica de la articulación de la rodilla. Murcia, Servicio de Radiología, Facultad de Medicina Universidad de Murcia, 2003.

Erbagci, H.; Gumusburun, E.; Bayram, M.; Karakurum, G. \& Sirikci, A. The normal menisci: in vivo MRI measurements. Surg. Radiol. Anat., 26(1):2832, 2004.

Fox, A. J. S.; Bedi, A. \& Rodeo, S. A. The basic science of human knee menisci: structure, composition, and function. Sports Health, 4(4):340-51, 2012.

Fox, A. J. S.; Wanivenhaus, F.; Burge, A. J.; Warren, R. F. \& Rodeo, S. A. The human meniscus: a review of anatomy, function, injury, and advances in treatment. Clin. Anat., 28(2):269-87, 2015.

Guo, H.; Santner, T. J.; Lerner, A. L. \& Maher, S. A. Reducing uncertainty when using knee-specific finite element models by assessing the effect of input parameters. J. Orthop. Res., 35(10):2233-42, 2017.
Jiang, D.; Luo, X.; Ao, Y.; Gong, X.; Wang, Y. J.; Wang, H. J.; Miao, Y.; Li, N. Zhang, J. Y. \& Yu, J. K. Risk of total/subtotal meniscectomy for respective medial and lateral meniscus injury: correlation with tear type, duration of complaint, age, gender and ACL rupture in 6034 Asian patients. BMC Surg., 17(1):127, 2017.

Klets, O.; Mononen, M. E.; Liukkonen, M. K.; Nevalainen, M. T.; Nieminen, M. T.; Saarakkala, S. \& Korhonen, R. K. Estimation of the effect of body weight on the development of osteoarthritis based on cumulative stresses in cartilage: data from the osteoarthritis initiative. Ann. Biomed. Eng., 46(2):334-44, 2018.

Kohn, M. D.; Sassoon, A. A. \& Fernando, N. D. Classifications in brief: KellgrenLawrence classification of osteoarthritis. Clin. Orthop. Relat. Res., 474(8):1886-93, 2016

Kontio, T.; Heliövaara, M.; Rissanen, H.; Knekt, P.; Aromaa, A. \& Solovieva, S. Risk factors for first hospitalization due to meniscal lesions - a populationbased cohort study with 30 years of follow-up. BMC Musculoskelet. Disord., 18(1):528, 2017.

Luczkiewicz, P.; Daszkiewicz, K.; Chrós'cielewski, J.; Witkowski, W. \& Winklewski, P. J. The influence of articular cartilage thickness reduction on meniscus biomechanics. PLoS One, 11(12):e0167733, 2016.

McDermott, I. D.; Sharifi, F.; Bull, A. M. J.; Gupte, C. M.; Thomas, R. W. \& Amis, A. A. An anatomical study of meniscal allograft sizing. Knee Surg. Sports Traumatol. Arthrosc., 12(2):130-5, 2004.

Orozco, G. A.; Tanska, P.; Mononen, M. E.; Halonen, K. S. \& Korhonen, R. K. The effect of constitutive representations and structural constituents of ligaments on knee joint mechanics. Sci. Rep., 8(1):2323, 2018.

Peters, A. E; Akhtar, R.; Comerford, E. J. \& Bates, K. T. Tissue material properties and computational modelling of the human tibiofemoral joint: a critical review. PeerJ, 6:e4298, 2018.

Shiang, T. \& Kotecha, H. CT diagnosis of bucket handle meniscus tear. Emerg. Radiol., 27:451-3, 2020.

Siorpaes, K.; Wenger, A.; Bloecker, K.; Wirth, W.; Hudelmaier, M. \& Eckstein, F. Interobserver reproducibility of quantitative meniscus analysis using coronal multiplanar DESS and IWTSE MR imaging. Magn. Reson. Med., 67(5):1419-26, 2012.

Smigielski, R.; Becker, R.; Zdanowicz, U. \& Ciszek, B. Medial meniscus anatomy-from basic science to treatment. Knee Surg. Sports Traumatol. Arthrosc., 23(1):8-14, 2015.

Sun, X.; Zhen, X.; Hu, X.; Li, Y.; Gu, S.; Gu, Y. \& Dong, H. Osteoarthritis in the middle-aged and elderly in China: prevalence and influencing factors. Int. J. Environ. Res. Public Health, 16(23):4701, 2019.

Torrez, J. C.; Olave, M. C.; Torrez, H. F. \& Olave, E. Biometric characteristics of knee meniscus in Chilean individuals. Int. J. Morphol., 29(3):1007-11, 2011.

Vélez, W.; Gómez, D. \& Thomson, P. Ajuste de modelos de elementos finitos: revisión. Dyna, 76(158):177-89, 2009.

Wenger, A.; Wirth, W.; Hudelmaier, M.; Noebauer-Huhmann, I.; Trattnig, S.; Bloecker, K.; Frobell, R. B.; Kwoh, C. K.; Eckstein, F. \& Englund, M. Meniscus body position, size, and shape in persons with and persons without radiographic knee osteoarthritis: quantitative analyses of knee magnetic resonance images from the osteoarthritis initiative. Arthritis Rheum., 65(7):1804-11, 2013.

Dirección para correspondencia:

Gloria Patricia Baena-Caldas, DDS, M.Sc.

Departamento de Morfología

Escuela de Ciencias Básicas

Facultad de Salud

Universidad del Valle

Calle 4B \# 36-00 Edificio 116

Cali - COLOMBIA

\section{Email: gloria.baena@correounivalle.edu.co} gpbaena@hotmail.com

Recibido : 19-07-2020

Aceptado: 08-09-2020 